\title{
First-order chemical reaction networks I: theoretical considerations
}

\author{
Roland Tóbiás $^{1}$ • László L. Stacho ${ }^{2}$ - Gyula Tasi ${ }^{1}$
}

Received: 21 February 2016 / Accepted: 4 June 2016

(C) Springer International Publishing Switzerland 2016

\begin{abstract}
Our former study Tóbiás and Tasi (J Math Chem 54:85, 2016) is continued, where a simple algebraic solution was given to the kinetic problem of triangle, quadrangle and pentangle reactions. In the present work, after defining chemical reaction networks and their connectedness, first-order chemical reaction networks (FCRNs) are studied on the basis of the results achieved by Chellaboina et al. (Control Syst 29:60, 2009). First, it is proved that an FCRN is disconnected iff its coefficient matrix is block diagonalizable. Furthermore, mass incompatibility is used to interpret the reducibility of subconservative networks. For conservative FCRNs, the so-called marker network is introduced, which is linearly conjugate to the original one, to describe the zero eigenvalue associated to the coefficient matrix of an FCRN. Instead of using graphtheoretical concepts, simple algebraic tools are applied to present and solve these problems. As an illustration, an industrially important ten-component (formal) FCRN is presented which has algebraically exact solution.
\end{abstract}

Keywords First-order reaction network - Algebraic model · Network decomposition · Mass incompatibility · Marker network · Multiplicity of the zero eigenvalue

\section{Introduction}

The graph-theoretical formalism of the chemical reaction networks (CRNs) was elaborated in the 1970s by Horn, Jackson and Feinberg [16,18,25] which has received

Gyula Tasi

tasi@chem.u-szeged.hu

1 Department of Applied and Environmental Chemistry, University of Szeged, Rerrich B. tér 1, Szeged 6720, Hungary

2 Bolyai Institute, University of Szeged, Aradi Vértanúk tere 1, Szeged 6720, Hungary 
permanent applications $[11,12,17,41]$. Within the framework of this theory, numerous new kinetic concepts (e.g. linkage class, stoichiometric compatibility class, weak reversibility, complex and detailed balanced network) were introduced and results of high interest (multistability [11,12], deficiency [17], global attractor [10] and persistency theorems [2,36], conjugacy of $C R N s[13,26,27])$ were achieved.

$C R N s$ can be defined not only in the language of graph theory. In the study published by Chellaboina et al. [7], matrix-vector notation is applied and the dynamical equations are set up in terms of vector-matrix exponentiation. This approach resulted in significant progress in the characterization of the solutions connected to the massbalance relations. As far as the algebraic treatment of the first-order linear systems of differential equations (FLSODE) is concerned, the books of Pontryagin [38] and Kailath [28] should be referred, where relevant information can be found about their structural properties, especially about their stability.

The qualitative theory of first-order reaction networks (FCRNs) was limited to the problems (stability [29], physical realizability [9], observability, controllability, identifiability [8], decomposability [34]) of compartmental systems [19,22,23], in chemical terms: isomerization reaction networks, IRNs. During the last two decades, the properties of arbitrary FCRNs have also been investigated in detail by Bernstein et al. $[4,5,7]$.

In this study, first we summarize the significant results of Chellaboina et al. [7] related to FCRNs and then we answer some further questions (e.g. mass incompatibility, multiplicity of the zero eigenvalue of the coefficient matrix). Instead of using graph-theoretical concepts, simple algebraic tools are applied to present and solve these problems. As an example, an industrially important ten-component (formal) $F C R N$ is presented which has an algebraically exact solution.

\section{Preliminaries}

\subsection{Algebraic model of $C R N s$}

Consider a homogeneous reaction system with $K$ chemical components and $R$ elementary reactions at constant temperature, pressure and volume according to the next scheme $[7,43]$ :

$$
\sum_{j=1}^{K} d_{i j} A_{j} \rightarrow \sum_{j=1}^{K} g_{i j} A_{j}\left(i \in \mathbb{Z}_{R}^{+}\right)
$$

where $d_{i j}$ and $g_{i j}$ are the left and right stoichiometric coefficients of the species $A_{j}$ in the $i$ th reaction. Equation (1) can be written with the matrices $\mathbf{D}=\left\{d_{i j}\right\}$ and $\mathbf{G}=\left\{g_{i j}\right\}$ as follows:

$$
\mathbf{D A} \rightarrow \text { GA }
$$

where $\mathbf{A}=\left\{A_{j}\right\}$ denotes the vector of the chemical components. In particular, regarding elementary reactions, $d_{i j}$ and $g_{i j}$ are nonnegative integers. If each of the reactions is of first-order, then $\mathbf{D}=\left\{\delta_{\eta_{i} j}\right\}$ where $\delta_{\eta_{i} j}$ stands for the Kronecker delta and there 
exists a unique $\eta_{i} \in \mathbb{Z}_{K}^{+}$for every $i \in \mathbb{Z}_{R}^{+}$. It is convenient to introduce the so-called stoichiometric matrix $\mathbf{S}=\left\{v_{i j}\right\}: \mathbf{S}=\mathbf{G}-\mathbf{D}$.

The time dependence of the concentration vector $\mathbf{C}=\left\{c_{j}\right\}$ is described with the following system of ordinary differential equations (mass-balance relations):

$$
\dot{\mathbf{C}}=\mathbf{S}^{T} \rho
$$

where $\dot{\mathbf{C}}=\left\{\dot{c}_{j}\right\}$ is the derivative of $\mathbf{C}$ with respect to time, $\boldsymbol{\rho}=\left\{r_{i}\right\}$ is the vector of the reaction rates, $\mathbf{S}^{T}$ is the transpose of $\mathbf{S}$. Reaction rates are approached via the law of kinetic mass-action [43]:

$$
r_{i}=k_{i} \prod_{l=1}^{K} c_{l}^{d_{i l}}
$$

where $k_{i}>0$ is the $i$ th elementary rate coefficient which depends on the temperature and, occasionally, on the pressure as well.

Assuming mass-action kinetics, the quadruple $\langle\mathbf{D}, \mathbf{G}, \mathbf{A}, \mathbf{k}\rangle$ is called the chemical reaction network $(C R N)$ if $\mathbf{k}=\left\{k_{i}\right\}$. For arbitrary permutation matrices $\mathbf{P}_{R} \in\{0,1\}^{R \times R}$ and $\mathbf{P}_{K} \in\{0,1\}^{K \times K},\langle\mathbf{D}, \mathbf{G}, \mathbf{A}, \mathbf{k}\rangle$ is equivalent to the network $\left\langle\mathbf{P}_{R} \mathbf{D P} \mathbf{P}_{K}, \mathbf{P}_{R} \mathbf{G} \mathbf{P}_{K}, \mathbf{P}_{K}^{T} \mathbf{A}, \mathbf{P}_{R} \mathbf{k}\right\rangle$.

To characterize the behavior of $C R N$ s concerning mass conservation, three important concepts [14] are necessary to mention. A network $\langle\mathbf{D}, \mathbf{G}, \mathbf{A}, \mathbf{k}\rangle$ is

- conservative if there exists $\mathbf{M} \in(0, \infty)^{K}$ such that $\mathbf{S M}=\mathbf{0}_{R}$ where $\mathbf{0}_{R}=\{0\}^{R}$;

- subconservative if there is an $\mathbf{M} \in(0, \infty)^{K}$ that $\mathbf{S M} \in(-\infty, 0]^{R}$;

- superconservative if $\mathbf{S M} \in[0, \infty)^{R}$ with an appropriate $\mathbf{M} \in(0, \infty)^{K}$.

\subsection{Decomposition of $C R N s$}

Consider the partitions $\mathcal{I}=\left\{\mathcal{I}_{k}: k \in \mathbb{Z}_{N_{C}}^{+}\right\}$and $\mathcal{J}=\left\{\mathcal{J}_{l}: l \in \mathbb{Z}_{N_{C}}^{+}\right\}$of $\mathbb{Z}_{R}^{+}$ and $\mathbb{Z}_{K}^{+}$where $N_{C}$ is the number of the partition cells. The couple $(\mathcal{I}, \mathcal{J})$ decomposes $\langle\mathbf{D}, \mathbf{G}, \mathbf{A}, \mathbf{k}\rangle$ if $d_{i j}=g_{i j}=0$ for $i \in \mathcal{I}_{k}$ and $j \in \mathcal{J}_{l}$ with $k \neq$ l. Suppose that the couples $(\mathcal{I}, \mathcal{J})$ and $\left(\mathcal{I}^{\prime}, \mathcal{J}^{\prime}\right)$ decompose $\langle\mathbf{D}, \mathbf{G}, \mathbf{A}, \mathbf{k}\rangle$ where $\mathcal{I}^{\prime}=\left\{\mathcal{I}_{l}^{\prime}: l \in \mathbb{Z}_{N_{C}^{\prime}}^{+}\right\}$and $\mathcal{J}^{\prime}=\left\{\mathcal{J}_{l}^{\prime}: l \in \mathbb{Z}_{N_{C}^{\prime}}^{+}\right\}$. Then $\left(\mathcal{I}^{\prime \prime}, \mathcal{J}^{\prime \prime}\right)$ with $\mathcal{I}^{\prime \prime}=$ $\left\{\mathcal{I}_{k} \cap \mathcal{I}_{l}^{\prime}: k \in \mathbb{Z}_{N_{C}}^{+} \wedge l \in \mathbb{Z}_{N_{C}^{\prime}}^{+} \wedge \mathcal{I}_{k} \cap \mathcal{I}_{l}^{\prime} \neq \varnothing\right\}$ and $\mathcal{J}^{\prime \prime}=\left\{\mathcal{J}_{k} \cap \mathcal{J}_{l}^{\prime}: k \in \mathbb{Z}_{N_{C}}^{+} \wedge l \in\right.$ $\left.\mathbb{Z}_{N_{C}^{\prime}}^{+} \wedge \mathcal{J}_{k} \cap \mathcal{J}_{l}^{\prime} \neq \varnothing\right\}$ does the same. Clearly, there is a finest decomposing couple of partitions $\left(\mathcal{I}^{*}, \mathcal{J}^{*}\right)$ with a maximal number of cells $N_{C}^{*}$ where $\mathcal{I}^{*}=\left\{\mathcal{I}_{l}^{*}: l \in \mathbb{Z}_{N_{C}^{*}}^{+}\right\}$ and $\mathcal{J}^{*}=\left\{\mathcal{J}_{l}^{*}: l \in \mathbb{Z}_{N_{C}^{*}}^{+}\right\}$. Notice, there are permutation matrices $\mathbf{P}_{R}$ and $\mathbf{P}_{K}$ block diagonalizing $\mathbf{D}$ and $\mathbf{G}$ :

$$
\begin{aligned}
& \mathbf{P}_{R} \mathbf{D P} \mathbf{P}_{K}=\operatorname{diag}_{i \in \mathbb{Z}_{N_{C}^{*}}^{+}}\left(\mathbf{D}_{i}\right) \\
& \mathbf{P}_{R} \mathbf{G P}_{K}=\operatorname{diag}_{i \in \mathbb{Z}_{N_{C}^{*}}^{+}}\left(\mathbf{G}_{i}\right)
\end{aligned}
$$


where $\mathbf{D}_{i}, \mathbf{G}_{i} \in \mathbb{R}^{R_{i} \times K_{i}}, \operatorname{diag}_{i \in \mathbb{Z}_{N_{C}^{*}}^{+}}\left(\mathbf{D}_{i}\right)=\operatorname{diag}\left(\mathbf{D}_{1}, \mathbf{D}_{2}, \ldots, \mathbf{D}_{N_{C}^{*}}\right), R_{i}$ and $K_{i}$ is the cardinality of $\mathcal{I}_{i}^{*}$ and $\mathcal{J}_{i}^{*}\left(i \in \mathbb{Z}_{N_{C}^{*}}\right)$. The matrices $\mathbf{P}_{R}$ and $\mathbf{P}_{K}$ partitionate the vectors $\mathbf{A}$ and $\mathbf{k}$, too:

$$
\begin{aligned}
\mathbf{P}_{R}^{T} \mathbf{A} & =\left(\begin{array}{llll}
\mathbf{A}_{1}^{T} & \mathbf{A}_{2}^{T} & \ldots & \mathbf{A}_{N_{C}^{*}}^{T}
\end{array}\right)^{T} \\
\mathbf{P}_{R} \mathbf{k} & =\left(\begin{array}{llll}
\mathbf{k}_{1}^{T} & \mathbf{k}_{2}^{T} & \ldots & \mathbf{k}_{N_{C}^{*}}^{T}
\end{array}\right)^{T}
\end{aligned}
$$

where $\mathbf{k}_{i}$ and $\mathbf{A}_{i}$ are of the type $R_{i} \times 1$ and $K_{i} \times 1$. The network $\left\langle\mathbf{D}_{i}, \mathbf{G}_{i}, \mathbf{A}_{i}, \mathbf{k}_{i}\right\rangle$ is called a maximally independent subnetwork of $\langle\mathbf{D}, \mathbf{G}, \mathbf{A}, \mathbf{k}\rangle$, and the set $\left\{\left\langle\mathbf{D}_{i}, \mathbf{G}_{i}, \mathbf{A}_{i}, \mathbf{k}_{i}\right\rangle\right.$ : $\left.i \in \mathbb{Z}_{N_{C}^{*}}^{+}\right\}$is a network decomposition of $\langle\mathbf{D}, \mathbf{G}, \mathbf{A}, \mathbf{k}\rangle$. A network is connected if $N_{C}^{*}=1$, otherwise, it is disconnected.

The matrices $\mathbf{D}$ and $\mathbf{G}$ can be written in the form of Eqs. (5) and (6) iff the matrix $\mathbf{H}=\mathbf{G}+\mathbf{D}$ is transformed by appropriate matrices $\mathbf{P}_{R}$ and $\mathbf{P}_{K}$ as follows:

$$
\mathbf{P}_{R} \mathbf{H} \mathbf{P}_{K}=\operatorname{diag}_{i \in \mathbb{Z}_{N_{C}^{*}}^{+}}\left(\mathbf{H}_{i}\right)
$$

where $\mathbf{H}_{i}=\mathbf{G}_{i}+\mathbf{D}_{i}$. If $N_{C}^{*} \neq 1$, then $\mathbf{H}$ is termed block diagonalizable. To determine $\mathbf{P}_{R}$ and $\mathbf{P}_{K}$ required by Eq. (9), the study of Schuster and Schuster [40] is worth citing.

\subsection{Dynamics of FCRNs}

Henceforward, first-order reaction networks (FCRNs) are examined exclusively. The network $\langle\mathbf{D}, \mathbf{G}, \mathbf{A}, \mathbf{k}\rangle$ is first-order if $\mathbf{D}=\left\{\delta_{\eta_{i} j}\right\}$. An FCRN is referred to as an isomerization reaction network $(I R N)$ if $\mathbf{G}=\left\{\delta_{\kappa_{i} j}\right\}$ where $\kappa_{i} \in \mathbb{Z}_{K}^{+}\left(i \in \mathbb{Z}_{R}^{+}\right)$. Regarding FCRNs, we get

$$
\dot{\mathbf{C}}=\mathbf{F C}
$$

where $\mathbf{F}=\left\{f_{j m}\right\}$ is the time-independent Jacobian of $\dot{\mathbf{C}}$ with respect to the vector C. $\mathbf{F}$ is called the coefficient matrix of FCRN which can also be expressed in the subsequent way [7]:

$$
\mathbf{F}=\mathbf{S}^{T} \operatorname{diag}(\mathbf{k}) \mathbf{D}
$$

where $\operatorname{diag}(\mathbf{k})=\operatorname{diag}\left(k_{1}, k_{2}, \ldots, k_{R}\right)$. The entry $f_{i j}$ can be written as follows:

$$
f_{i j}=\sum_{l=1}^{R} \sum_{m=1}^{R} v_{l i} \delta_{l m} k_{l} \delta_{\eta_{m} j}=\sum_{m=1}^{R} v_{m i} \delta_{\eta_{m} j} k_{m} .
$$

Since $v_{m i}=g_{m i}-\delta_{\eta_{m} i}$, the diagonal and off-diagonal entries need to be calculated separately: 


$$
f_{i j}= \begin{cases}\sum_{m=1}^{R}\left(g_{m i}-1\right) k_{m}, & \text { if } i=j \\ \eta_{m}=i & \\ \sum_{m=1}^{R} g_{m i} k_{m}, & \text { otherwise. } \\ \eta_{m}=j & \end{cases}
$$

Equation (13) clearly shows that the relation $f_{i j} \geq 0$ implies if $i \neq j$, therefore $\mathbf{F}$ is a Metzlerian [7].

Equation (10) is a FLSODE with initial condition $\mathbf{C}_{0}=\mathbf{C}(0)=\left\{c_{0 j}\right\}$ whose solution is

$$
\mathbf{C}(t)=\sum_{k=0}^{\infty} \frac{t^{k}}{k !}\left(\frac{d^{(k)} \mathbf{C}(t)}{d t^{k}}\right)_{t=0}=\sum_{k=0}^{\infty} \frac{t^{k}}{k !} \mathbf{F}^{k} \mathbf{C}_{0}=\exp (\mathbf{F} t) \mathbf{C}_{0}
$$

where the matrix $\exp (\mathbf{F} t)$ is used [30,31]. After linear algebraic transformations [32, 43], we get

$$
\mathbf{C}(t)=\mathbf{F}_{\mathbf{C}_{0}} \mathbf{V}^{-1} \mathbf{E}(t)
$$

where

$$
\begin{aligned}
\mathbf{F}_{\mathbf{C}_{0}} & =\left\{\mathbf{C}_{0}, \mathbf{F C}_{0}, \mathbf{F}^{2} \mathbf{C}_{0}, \ldots, \mathbf{F}^{K-1} \mathbf{C}_{0}\right\} \\
\mathbf{E}(t) & =\left\{e^{\lambda_{1} t}, t e^{\lambda_{1} t}, t^{2} e^{\lambda_{1} t}, \ldots, t^{\mu_{1}-1} e^{\lambda_{1} t}, \ldots, e^{\lambda_{L} t}, t e^{\lambda_{L} t}, t^{2} e^{\lambda_{L} t}, \ldots, t^{\mu_{L}-1} e^{\lambda_{L} t}\right\}^{T} \\
\mathbf{V} & =\left\{\mathbf{E}(0), \dot{\mathbf{E}}(0), \ddot{\mathbf{E}}(0), \ldots, \mathbf{E}^{(K-1)}(0)\right\} .
\end{aligned}
$$

$\mathbf{F}_{\mathbf{C}_{0}}, \mathbf{E}(t)$ and $\mathbf{V}$ in Eqs. (16)-(18) are the Krylov matrix, the time evolution vector and the Vandermonde matrix, respectively. The parameter $L$ is the number of distinct eigenvalues of $\mathbf{F}, \lambda_{k}$ is the $k$ th eigenvalue, $\mu_{k}$ is its multiplicity $\left(k \in \mathbb{Z}_{L}^{+}\right)$. The columns of $\mathbf{V}$ correspond to the various derivatives of the vector $\mathbf{E}(t)$ taken at $t=0$. If the eigenvalues of the matrix $\mathbf{F}$ are calculated numerically, Eq. (15) is called the semianalytical solution of Eq. (10).

Notice, that several algorithms (e.g. classical integration [24], transfer function [1,33], matrix and convolution [37] methods) can be used for solving Eq. (10).

\subsection{Nonnegativity and semistable nonnegative equilibria}

Since the entries of $\mathbf{C}(t)$ represent the concentrations belonging to the species of an $F C R N$, therefore $\mathbf{C}(t) \in[0, \infty)^{K}$ (nonnegativity) and $\mathbf{C}_{\infty} \in[0, \infty)^{K}$ (semistable nonnegative equilibrium) are indubitable for all $t \geq 0$ where $\mathbf{C}_{\infty}=\lim _{t \rightarrow \infty} \mathbf{C}(t)$. Furthermore, based on [7], the nonnegativity and stability of Eq. (14) are investigated. 
(The analysis of nonnegativity concerning to arbitrary $C R N$ s can be also found in the work by Volpert [44].)

Nonnegativity of the function $\mathbf{C}(t)=\exp (\mathbf{F} t) \mathbf{C}_{0}$ implies from the well-known theorem that $\exp (\mathbf{F} t) \in[0, \infty)^{K \times K}$ is valid for all $t \geq 0$ iff $\mathbf{F}$ is a Metzler matrix [20]. As this is fulfilled by Eq. (13), so $\exp (\mathbf{F} t) \mathbf{C}_{0} \in[0, \infty)^{K}$ if $\mathbf{C}_{0} \in[0, \infty)^{K}$.

To examine the stability behavior of Eq. (14), some notions need to be recalled. The vector $\tilde{\mathbf{C}}$ is an equilibrium point if $\mathbf{F} \tilde{\mathbf{C}}=\mathbf{0}_{K} \cdot \tilde{\mathbf{C}}$ is Lyapunov stable if there exists a $\theta>0$ for all $t \geq 0$ and $v>0$ such that $\|\mathbf{C}-\tilde{\mathbf{C}}\|<v$ if $\left\|\tilde{\mathbf{C}}-\mathbf{C}_{0}\right\|<\theta$ where || || denotes the Eucledian norm. A Lyapunov-stabile point $\tilde{\mathbf{C}}$ is regarded semistable if there is $v>0$ such that $\left\|\tilde{\mathbf{C}}-\mathbf{C}_{0}\right\|<v$ involves Lyapunov stability of the vector $\mathbf{C}_{\infty}$ for every $\mathbf{C}_{0}$. It is proved [3] that $\tilde{\mathbf{C}}$ is semistable iff each eigenvalue of $\mathbf{F}$ has a negative real part or is zero and the number of linearly independent eigenvectors related to the zero eigenvalue is equal to its (algebraic) multiplicity. A sufficient condition can also be derived [20]: if $\mathbf{F}^{T} \mathbf{M} \in(-\infty, 0]^{K}$ for $\mathbf{M} \in(0, \infty)^{K}$, then $\tilde{\mathbf{C}}=\mathbf{0}_{K}$ is a semistable equilibrium point of Eq. (10). This constraint is fulfilled by subconservative systems because

$$
\mathbf{F}^{T} \mathbf{M}=\left(\mathbf{S}^{T} \operatorname{diag}(\mathbf{k}) \mathbf{D}\right)^{T} \mathbf{M}=\mathbf{D}^{T} \operatorname{diag}(\mathbf{k}) \mathbf{S M}
$$

where $\mathbf{S M} \in(-\infty, 0]^{R}$, consequently $\mathbf{F}^{T} \mathbf{M} \in(-\infty, 0]^{K}$. Notice, that the vector $\mathbf{M}$ can be replaced by the vector of molar masses; therefore, if these quantities are available, there is no need to search for such a vector.

\section{Results}

\subsection{Relation of block diagonalizability of $\mathbf{F}$ and $\mathbf{H}$}

In this section it is revealed that the following two predicates are equivalent for FCRNs: $\mathcal{A}) \mathbf{H}$ is block diagonalizable, $\mathcal{B}) \mathbf{F}$ is block diagonalizable.

Proof of $\mathcal{A}) \Rightarrow \mathcal{B}$ ) Suppose that $\mathbf{P}_{R}$ and $\mathbf{P}_{K}$ transform $\mathbf{H}$ into block diagonal form in terms of Eq. (9). Then both $\mathbf{D}$ and $\mathbf{G}$ are block diagonal and diag (k) is partitioned into blocks by the permutation matrix $\mathbf{P}_{R}$ :

$$
\mathbf{P}_{R} \operatorname{diag}(\mathbf{k}) \mathbf{P}_{R}^{T}=\operatorname{diag}_{i \in \mathbb{Z}_{N_{C}^{*}}^{+}}\left(\operatorname{diag}\left(\mathbf{k}_{i}\right)\right)
$$

Adapting these considerations,

$$
\mathbf{F}=\operatorname{diag}_{i \in \mathbb{Z}_{N_{C}^{*}}^{+}}\left(\mathbf{S}_{i}\right)^{T} \operatorname{diag}_{i \in \mathbb{Z}_{N_{C}^{*}}^{+}}\left(\operatorname{diag}\left(\mathbf{k}_{i}\right)\right) \operatorname{diag}_{i \in \mathbb{Z}_{N_{C}^{*}}^{+}}\left(\mathbf{D}_{i}\right)
$$

where $\mathbf{S}_{i}=\mathbf{G}_{i}-\mathbf{D}_{i}$. In this expression, the matrix $\boldsymbol{\Phi}_{i}=\mathbf{S}_{i}^{T} \operatorname{diag}\left(\mathbf{k}_{i}\right) \mathbf{D}_{i}$ can be utilized which yields $\mathbf{F}=\operatorname{diag}_{i \in \mathbb{Z}_{N_{C}^{*}}^{+}}\left(\boldsymbol{\Phi}_{i}\right)$, therefore $\left.\left.\mathcal{A}\right) \Rightarrow \mathcal{B}\right)$. 
Proof of $\mathcal{B}) \Rightarrow \mathcal{A}$ ). From Eq. (13), it follows that $f_{i j}$ vanishes only if each term of the sum is identically zero as a result of $g_{m i} \geq 0$ and $k_{m}>0$. This means that $g_{m i}=0$ comes from $f_{i j}=0$ and $\eta_{m}=j$, i.e. $A_{j}$ is not converted into $A_{i} ; f_{i j}=$ $f_{j i}=0$ implicates that $A_{i}$ and $A_{j}$ do not take part in the same reaction. Let $\mathbf{F}=$ $\operatorname{diag}_{i \in \mathbb{Z}_{N_{C}^{*}}^{+}}\left(\boldsymbol{\Phi}_{i}\right)\left(\boldsymbol{\Phi}_{i} \in \mathbb{R}^{K_{i} \times K_{i}} ; i \in \mathbb{Z}_{N_{C}^{*}}^{+}\right)$be considered. In this respect, $\mathbf{A}$ can be partitioned by a permutation matrix $\mathbf{P}_{K}=\left\{\delta_{i j}\right\}$ in the form of Eq. (7). Since $\mathbf{F}$ is a block diagonal matrix, the species belonging to $\mathbf{A}_{i}$ and $\mathbf{A}_{j}$ do not participate in the same conversion $\left(i \neq j ; i, j \in \mathbb{Z}_{N_{C}^{*}}^{+}\right.$). There needs to be such a permutation matrix $\mathbf{P}_{R}$ which block diagonalizes $\mathbf{D}$ :

$$
\mathbf{P}_{R} \mathbf{D}=\operatorname{diag}_{i \in \mathbb{Z}_{N_{C}^{*}}^{+}}\left(\mathbf{D}_{i}\right)
$$

where $\mathbf{D}_{i} \in\{0,1\}^{R_{i} \times K_{i}}$ and $R_{i}$ is the number of reactions taking place among the species of $\mathbf{A}_{i}\left(i \in \mathbb{Z}_{N_{C}^{*}}^{+}\right)$. This permutation $\mathbf{P}_{R}$ has an impact on the shape of the matrix G:

$$
\mathbf{P}_{R} \mathbf{G}=\left(\begin{array}{llll}
\boldsymbol{\Gamma}_{1}^{T} & \boldsymbol{\Gamma}_{2}^{T} & \ldots & \boldsymbol{\Gamma}_{N_{C}^{*}}^{T}
\end{array}\right)^{T}
$$

where $\boldsymbol{\Gamma}_{i} \in[0, \infty)^{R_{i} \times K}$. Let $\boldsymbol{\Gamma}_{i}$ be partitioned into the subsequent form:

$$
\boldsymbol{\Gamma}_{i}=\left(\begin{array}{llll}
\mathbf{G}_{i 1} & \mathbf{G}_{i 2} & \ldots & \mathbf{G}_{i N_{C}^{*}}
\end{array}\right)
$$

where $\mathbf{G}_{i j}=[0, \infty)^{R_{i} \times K_{j}}\left(i, j \in \mathbb{Z}_{N_{C}^{*}}^{+}\right)$. With the help of Eqs. (22) and (24), $\mathbf{P}_{R} \mathbf{G}$ can be written as follows:

$$
\mathbf{P}_{R} \mathbf{G}=\left(\begin{array}{cccc}
\mathbf{G}_{11} & \mathbf{G}_{12} & \ldots & \mathbf{G}_{1 N_{C}^{*}} \\
\mathbf{G}_{21} & \mathbf{G}_{22} & \ldots & \mathbf{G}_{2 N_{C}^{*}} \\
\vdots & \vdots & \ddots & \vdots \\
\mathbf{G}_{N_{C}^{*} 1} & \mathbf{G}_{N_{C}^{*} 2} & \ldots & \mathbf{G}_{N_{C}^{*} N_{C}^{*}}
\end{array}\right)
$$

However, $\mathbf{G}_{i j}=\mathbf{0}_{K_{i} \times K_{j}}\left(\mathbf{0}_{K_{i} \times K_{j}}=\{0\}^{K_{i} \times K_{j}} ; i \neq j\right)$ is caused by the block diagonality of $\mathbf{F}$, in one word, $\mathbf{P}_{R} \mathbf{G}=\operatorname{diag}_{i \in \mathbb{Z}_{N_{C}^{*}}^{+}}\left(\mathbf{G}_{i i}\right)$. In this instance, $\mathbf{P}_{R} \mathbf{H}$ is block diagonal, i.e. $\mathcal{B}) \Rightarrow \mathcal{A}$ ). Our further statements are drawn up for connected FCRNs, namely, the matrix $\mathbf{F}$ is not deemed to be block diagonalizable.

\subsection{Mass incompatibility in subconservative systems}

Recall from Sect. 2.4 that subconservative systems exhibit semistable behavior. Supposing a nonnegative integer-type matrix G, subconservativity implies certain restrictions on the kinetics of the network $\langle\mathbf{D}, \mathbf{G}, \mathbf{A}, \mathbf{k}\rangle$. It is enough to mention, the species $A_{i}$ cannot be converted into $A_{j}$ if $M_{i}<M_{j}$ (mass incompatibility). In the sequel, we investigate the consequences of the condition $\mathbf{S M} \in(-\infty, 0]^{R}$. The $m$ th entry of the vector $\mathbf{S M}$ is written as follows: 


$$
\langle\mathbf{S M}\rangle_{m}=\langle\mathbf{G M}\rangle_{m}-\langle\mathbf{D M}\rangle_{m}=\sum_{j=1}^{K} g_{m j} M_{j}-M_{\eta_{m}}
$$

On the basis of the relation between $M_{j}$ and $M_{\eta_{m}},\langle\mathbf{G M}\rangle_{m}$ is divided into three terms:

$$
\langle\mathbf{S M}\rangle_{m}=\sum_{\substack{j \in \mathbb{Z}_{K}^{+} \\ M_{j}<M_{\eta_{m}}}} g_{m j} M_{j}+M_{\eta_{m}} \sum_{\substack{j \in \mathbb{Z}_{K}^{+} \\ M_{j}=M_{\eta_{m}}}} g_{m j}+\sum_{\substack{j \in \mathbb{Z}_{K}^{+} \\ M_{j}>M_{\eta_{m}}}} g_{m j} M_{j}-M_{\eta_{m}} .
$$

Since $\langle\mathbf{S M}\rangle_{m} \leq 0$, the entry $\langle\mathbf{S M}\rangle_{m}$ can be estimated from below by neglecting the terms related to $M_{j}<M_{\eta_{m}}$ and $M_{j}=M_{\eta_{m}}$ :

$$
0 \geq\langle\mathbf{S M}\rangle_{m} \geq \sum_{\substack{j \in \mathbb{Z}_{K}^{+} \\ M_{j}>M_{\eta_{m}}}} g_{m j} M_{j}-M_{\eta_{m}} \geq M_{m}^{\otimes} \sum_{\substack{j \in \mathbb{Z}_{K}^{+} \\ M_{j}>M_{\eta_{m}}}} g_{m j}-M_{\eta_{m}}
$$

where $M_{m}^{\otimes}=\min _{\substack{j \in \mathbb{Z}_{K^{+}} \\ M_{j}>M_{\eta_{m}}}} M_{j}$. Rearranging Eq. (28), we obtain

$$
\sum_{\substack{j \in \mathbb{Z}_{K}^{+} \\ M_{j}>M_{\eta_{m}}}} g_{m j} \leq \frac{M_{\eta_{m}}}{M_{m}^{\otimes}}<1
$$

where $M_{\eta_{m}} / M_{m}^{\otimes}<1$. Since the entries of $\mathbf{G}$ are nonnegative integers, we have $g_{m j}=0$ for $M_{j}>M_{\eta_{m}}$, establishing mass incompatibility. Based on all these, we get

$$
\langle\mathbf{S M}\rangle_{m}=\sum_{\substack{j \in \mathbb{Z}_{K}^{+} \\ M_{j}<M_{\eta_{m}}}} g_{m j} M_{j}+M_{\eta_{m}} \sum_{\substack{j \in \mathbb{Z}_{K}^{+} \\ M_{j}=M_{\eta_{m}}}} g_{m j}-M_{\eta_{m}}
$$

Estimating $\langle\mathbf{S} \boldsymbol{\mu}\rangle_{m}$ from below and simplifying by $M_{\eta_{m}}$, we conclude

$$
1 \geq \sum_{\substack{j \in \mathbb{Z}_{K}^{+} \\ M_{j}=M_{\eta_{m}}}} g_{m j}
$$

This can be interpreted as at most one species $A_{j}$ is produced in each reaction with the mass $M_{j}=M_{\eta_{m}}$, involving $g_{m j}=1$. Assuming that $M_{l}=M_{\eta_{m}}$ and $g_{m l}=1$ for some $l \in \mathbb{Z}_{K}^{+}$, due to Eq. (30), we acquire

$$
0 \geq \sum_{\substack{j \in \mathbb{Z}_{K}^{+} \\ M_{j}<M_{\eta_{m}}}} g_{m j} M_{j}+M_{\eta_{m}}-M_{\eta_{m}}=\sum_{\substack{j \in \mathbb{Z}_{K}^{+} \\ M_{j}<M_{\eta_{m}}}} g_{m j} M_{j} .
$$


In other words, $g_{m j}=0$ for every $j \in \mathbb{Z}_{K}^{+}$in case of $M_{j}>M_{\eta_{m}}$.

Mass incompatibility affects not only $\mathbf{G}$ but also $\mathbf{F}$. It is easily conceded that $f_{i j}=0$ if $M_{i}>M_{j}\left(i, j \in \mathbb{Z}_{K}^{+}\right)$: since $A_{j}$ is not transformed into $A_{i}$, hence every $g_{m j}$ is equal to zero in Eq. (13), i.e. $f_{i j}=0$. Consequently, permuting the chemical components in accordance with decreasing order of their masses, a lower block triangular matrix is received:

$$
\mathbf{F}=\left(\begin{array}{cccc}
\mathbf{F}_{11} & & & \\
\mathbf{F}_{21} & \mathbf{F}_{22} & & \\
\vdots & \vdots & \ddots & \\
\mathbf{F}_{N_{W}} & \mathbf{F}_{N_{W}} & \ldots & \mathbf{F}_{N_{W} N_{W}}
\end{array}\right)
$$

where $N_{W}$ is the number of species with different masses and the blocks explicitly not marked are zeros. This form is really advantageous because the eigenvalues of $\mathbf{F}$ are identical to the ones of the matrices $\mathbf{F}_{i i}$ with significantly smaller size $\left(i \in \mathbb{Z}_{N_{W}}^{+}\right)$. The role of block triangularity is expounded in more detail in Sect. 3.4.

\subsection{Concept of the marker network}

Most chemical processes are studied in a closed system therefore paying particular attention to conservative $F C R N$ s is advisable. In this section it is explored how to transform a conservative network into a (formal) network containing only isomerization reaction steps.

Equation (19) clearly shows that $\mathbf{F}^{T} \mathbf{M}=\mathbf{0}_{K}$ is a consequence of the criterion $\mathbf{S M}=\mathbf{0}_{R}$. Introducing the "all-onces vector" $\mathbf{1}_{K} \in\{1\}^{K}$, we can write

$$
\mathbf{F}^{T} \mathbf{M}=\mathbf{F}^{T} \operatorname{diag}(\mathbf{M}) \mathbf{1}_{K}=\mathbf{0}_{K}
$$

Equation (34) is invariant under the multiplication with an arbitrary matrix, therefore

$$
\operatorname{diag}^{-1}(\mathbf{M}) \mathbf{F}^{T} \operatorname{diag}(\mathbf{M}) \mathbf{1}_{K}=\left(\operatorname{diag}(\mathbf{M}) \mathbf{F} \operatorname{diag}^{-1}(\mathbf{M})\right)^{T} \mathbf{1}_{K}=\mathbf{F}^{\prime T} \mathbf{1}_{K}=\mathbf{0}_{K}
$$

where $\operatorname{diag}^{-1}(\mathbf{M})=\operatorname{diag}\left(M_{1}^{-1}, M_{2}^{-1}, \ldots, M_{K}^{-1}\right)$ and $\mathbf{F}^{\prime}=\operatorname{diag}(\mathbf{M}) \mathbf{F d i a g}{ }^{-1}(\mathbf{M})$. It follows that

$$
f_{i j}^{\prime}=\sum_{k=1}^{K} \sum_{l=1}^{K} \delta_{i k} M_{i} f_{k l} \delta_{l j} M_{l}^{-1}=\frac{M_{i}}{M_{j}} f_{i j}\left(i \in \mathbb{Z}_{K}^{+}\right)
$$

Since $f_{i j} \geq 0$, therefore $f_{i j}^{\prime} \geq 0(i \neq j)$, and the diagonal entries $f_{i i}^{\prime}$ can be determined in the following way [see Eq. (35)]: 


$$
f_{i i}^{\prime}=-\sum_{\substack{k=1 \\ k \neq i}}^{K} f_{k i}^{\prime}
$$

Equation (35) suggests, there is a (formal) $I R N\left\langle\left\{\delta_{\eta_{l}^{\prime} j}\right\},\left\{\delta_{\kappa_{l}^{\prime} j}\right\},\left\{A_{j}^{\prime}\right\},\left\{k_{l}^{\prime}\right\}\right\rangle$ with the coefficient matrix $\mathbf{F}^{\prime}$ where the mass of the species $A_{j}^{\prime}$ is equal to one $\left(\eta_{l}^{\prime}, \kappa_{l}^{\prime} \in\right.$ $\left.\mathbb{Z}_{K}^{+} ; l \in \mathbb{Z}_{R^{\prime}}^{+}\right)$. Let us attempt to define such a network. At first, the following constraints are considered: $\eta_{k}^{\prime}=\eta_{l}^{\prime} \Rightarrow \kappa_{k}^{\prime} \neq \kappa_{l}^{\prime} ; \kappa_{k}^{\prime}=\kappa_{l}^{\prime} \Rightarrow \eta_{k}^{\prime} \neq \eta_{l}^{\prime} ; \eta_{k}^{\prime} \neq \kappa_{k}^{\prime}\left(k \neq l ; k, l \in \mathbb{Z}_{R^{\prime}}^{+}\right)$ excluding „self reactions" and ,parallel reactions" from $A_{\eta_{l}^{\prime}}^{\prime}$ into $A_{\kappa_{l}^{\prime}}^{\prime}$. Let $R^{\prime}$ be the number of nonzero off-diagonal entries in the matrix $\mathbf{F}^{\prime}$, and $k_{l}^{\prime}=f_{\kappa_{l}^{\prime} \eta_{l}^{\prime}}^{\prime} \neq 0$. This network contains $A_{i}^{\prime} \rightarrow A_{j}^{\prime}$ with the rate coefficient $f_{j i}^{\prime}$ iff $f_{j i}^{\prime} \neq 0(i \neq j)$.

In this context, $\left\langle\mathbf{D}^{\prime}, \mathbf{G}^{\prime}, \mathbf{A}^{\prime}, \mathbf{k}^{\prime}\right\rangle$ is referred to as the marker network of $\langle\mathbf{D}, \mathbf{G}, \mathbf{A}, \mathbf{k}\rangle$ if $\mathbf{D}^{\prime}=\left\{\delta_{\eta_{l}^{\prime} j}\right\}, \mathbf{G}^{\prime}=\left\{\delta_{\kappa_{l}^{\prime} j}\right\}, \mathbf{A}^{\prime}=\left\{A_{j}^{\prime}\right\}$ and $\mathbf{k}^{\prime}=\left\{k_{l}^{\prime}\right\}$. Next we prove that $\mathbf{F}^{\prime}=$ $\left(\mathbf{G}^{\prime}-\mathbf{D}^{\prime}\right)^{T} \operatorname{diag}\left(\mathbf{k}^{\prime}\right) \mathbf{D}^{\prime}$. Denoting the matrix $\left(\mathbf{G}^{\prime}-\mathbf{D}^{\prime}\right)^{T} \operatorname{diag}\left(\mathbf{k}^{\prime}\right) \mathbf{D}^{\prime}$ by $\tilde{\mathbf{F}}=\left\{\tilde{f}_{i j}\right\}$, off-diagonal entries $\tilde{f}_{i j}$ can be given

$$
\tilde{f}_{i j}=\sum_{\substack{m=1 \\ \eta_{m}^{\prime}=j}}^{R^{\prime}} \delta_{\kappa_{m}^{\prime} i} f_{\kappa_{m}^{\prime} \eta_{m}^{\prime}}^{\prime}=\sum_{\substack{m=1 \\ \eta_{m}^{\prime}=j}}^{R^{\prime}} \delta_{\kappa_{m}^{\prime} i} f_{\kappa_{m}^{\prime} j}^{\prime},
$$

in agreement with Eq. (13). Obviously $f_{\kappa_{m}^{\prime} j}^{\prime} \neq 0$ is fulfilled just in case of $\kappa_{m}^{\prime}=i$. Only a unique index $m \in \mathbb{Z}_{R^{\prime}}^{+}$has this property, thus $\tilde{f}_{i j}=f_{i j}^{\prime}$. Using Eq. (38):

$$
\tilde{f}_{i i}=\sum_{\substack{m=1 \\ \eta_{m}=i}}^{R}\left(\delta_{\kappa_{m}^{\prime} i}-1\right) f_{\kappa_{m}^{\prime} \eta_{m}^{\prime}}^{\prime}=-\sum_{\substack{m=1 \\ \eta_{m}^{\prime}=i}}^{R^{\prime}} f_{\kappa_{m}^{\prime} i}^{\prime}
$$

where $\delta_{\kappa_{m}^{\prime} i}=0$ is applied at the hand of $\eta_{m}^{\prime} \neq \kappa_{m}^{\prime}$. Regarding the case $\eta_{m}^{\prime}=i$, summation needs to be performed on the row indices of nonzero off-diagonal entries related to the $i$ th column of $\mathbf{F}^{\prime}$ :

$$
\tilde{f}_{i i}=-\sum_{\substack{k=1 \\ k \neq i ; \tilde{f}_{k i} \neq 0}}^{K} f_{k i}^{\prime}=-\sum_{\substack{k=1 \\ k \neq i}}^{K} f_{k i}^{\prime}=f_{i i}^{\prime}
$$

It can be shown that $\mathbf{C}^{\prime}=\operatorname{diag}(\mathbf{M}) \mathbf{C}$ where $\mathbf{C}^{\prime}$ is the concentration vector associated to the vector $\mathbf{A}^{\prime}$. For this purpose, we need to construct the subsequent system of equations:

$$
\dot{\mathbf{C}}^{\prime}=\mathbf{F}^{\prime} \mathbf{C}^{\prime}
$$


Multiplying Eq. (10) by diag (M), Eq. (41) is obtained, since

$$
\operatorname{diag}(\mathbf{M}) \dot{\mathbf{C}}=\operatorname{diag}(\mathbf{M}) \mathbf{F}\left[\operatorname{diag}^{-1}(\mathbf{M}) \operatorname{diag}(\mathbf{M})\right] \mathbf{C}=\mathbf{F}^{\prime} \operatorname{diag}(\mathbf{M}) \mathbf{C} .
$$

Moreover, the relation $\mathbf{C}^{\prime}=\operatorname{diag}(\mathbf{M}) \mathbf{C}$ establishes a linear conjugacy relationship [26] between the vectors $\mathbf{C}$ and $\mathbf{C}^{\prime}$, i.e. $\left\langle\mathbf{D}^{\prime}, \mathbf{G}^{\prime}, \mathbf{A}^{\prime}, \mathbf{k}^{\prime}\right\rangle$ is a linearly conjugate network of $\langle\mathbf{D}, \mathbf{G}, \mathbf{A}, \mathbf{k}\rangle$.

Based on all these, it is evident that the network $\left\langle\mathbf{D}^{\prime}, \mathbf{G}^{\prime}, \mathbf{A}^{\prime}, \mathbf{k}^{\prime}\right\rangle$ indicates the dynamic behavior of $\langle\mathbf{D}, \mathbf{G}, \mathbf{A}, \mathbf{k}\rangle$, thus in certain cases (for instance, in characterizing the eigenvalues of the matrix $\mathbf{F}$ ) examining the properties of the marker network may be sufficient.

\subsection{Multiplicity of the zero eigenvalue in conservative $F C R N \mathrm{~s}$}

As presented for subconservative FCRNs in Sect. 3.2, if the molar masses of the species are not equal, an appropriate permutation matrix $\mathbf{P}_{K}$ transforms the matrix $\mathbf{F}$ into a lower block triangular form. In conservative systems, structure of Eq. (33) can be refined, furthermore, it can be derived under what conditions a diagonal block has a zero eigenvalue.

Knowing that $\mathbf{F}^{\prime}$ is a Metzlerian with the effect of $\mathbf{F}^{\prime T} \mathbf{1}_{K}=\mathbf{0}_{K}$, it is corresponded to the Laplacian of a simple weighted digraph, accordingly, the singularity of the diagonal blocks can be handled on the basis of graph theoretical principles [35]. In this section, recalling the works by Hearon and Taussky [22,23,42], we follow a path along which the problem of the zero eigenvalue can be studied purely with algebraic tools. Before, some linear algebraic notions need to be introduced.

The matrix $\mathbf{Q}=\left\{q_{i j}\right\}\left(\mathbf{Q} \in \mathbb{C}^{K \times K}\right)$ is irreducible [6] if $K=1$ or for each partition $\left\{\mathcal{U}, \mathcal{U}^{\prime}\right\}$ of $\mathbb{Z}_{K}^{+}\left(\mathcal{U}, \mathcal{U}^{\prime} \neq \varnothing\right)$ there exist $u \in \mathcal{U}$ and $u^{\prime} \in \mathcal{U}^{\prime}$ such that $q_{u u^{\prime}} \neq 0 . \mathbf{Q}$ is reducible if it is not irreducible. Notice, that if a matrix $\mathbf{Q} \in[0, \infty)^{K \times K}$ is irreducible, then $\max _{i=1}^{K}\left|\omega_{i}\right|$ is a simple eigenvalue where $\omega_{i}$ denotes the $i$ th eigenvalue of $\mathbf{Q}(i \in$ $\mathbb{Z}_{K}^{+}$) (Perron-Frobenius theorem [6]). In case of $K>1, \mathbf{Q}$ is reducible iff it can be written in the next form:

$$
\mathbf{P}_{K} \mathbf{Q P}_{K}^{T}=\left(\begin{array}{cccc}
\mathbf{Q}_{11} & & & \\
\mathbf{Q}_{21} & \mathbf{Q}_{22} & & \\
\vdots & \vdots & \ddots & \\
\mathbf{Q}_{n_{T} 1} & \mathbf{Q}_{n_{T} 2} & \ldots & \mathbf{Q}_{n_{T} n_{T}}
\end{array}\right)
$$

where $\mathbf{P}_{K}$ is a suitable permutation matrix and $n_{T}>1$. If $n_{T} \geq 1$ and the blocks $\mathbf{Q}_{i i}$ are irreducible, the matrix $\mathbf{P}_{K} \mathbf{Q} \mathbf{P}_{K}^{T}$ is called the Frobenius normal form [21] of the matrix $\mathbf{Q}$ which can be determined by several methods (e.g. Tarjan algorithm [39]).

Later we also use a theorem proved by Taussky [42]: if $\mathbf{Q} \in \mathbb{R}^{K \times K}$ is an irreducible Metzlerian and $-q_{i i} \geq \sum_{j=1, j \neq i}^{K} q_{j i}\left(i \in \mathbb{Z}_{K}^{+}\right)$, then $\operatorname{det}(\mathbf{Q})=0$ is equivalent to the equation $\mathbf{Q}^{T} \mathbf{1}_{K}=\mathbf{0}_{K}$. (Although Taussky stated this theorem for the matrix $-\mathbf{Q}$, the change of sign above is trivial, which is justified by the further considerations.) 
Based on the Pearron-Frobenius theorem, it is also shown by Hearon [23] that the zero eigenvalue is simple for every irreducible Metzlerian $\mathbf{Q} \in \mathbb{R}^{K \times K}$ with the property of $\mathbf{Q}^{T} \mathbf{1}_{K}=\mathbf{0}_{K}$.

After this brief algebraic overview, the characterization of $\mathbf{F}^{\prime}$ is continued. Since $\mathbf{F}^{\prime}$ is a Metzlerian with the relation $\mathbf{F}^{\prime T} \mathbf{1}_{K}=\mathbf{0}_{K}$, the conditions of Taussky's theorem (aside from the irreducibility) are fulfilled. We state that

$$
\begin{aligned}
& \mathbf{P}_{K} \mathbf{F}^{\prime} \mathbf{P}_{K}^{T} \mathbf{1}_{K}=\mathbf{0}_{K} \\
& \left\langle\mathbf{P}_{K} \mathbf{F}^{\prime} \mathbf{P}_{K}^{T}\right\rangle_{i j} \geq 0\left(i \neq j ; i, j \in \mathbb{Z}_{K}^{+}\right)
\end{aligned}
$$

where $\mathbf{P}_{K}$ is an arbitrary permutation matrix. To prove Eq. (44), consider the following equation:

$$
\mathbf{F}^{\prime T} \mathbf{1}_{K}=\mathbf{F}^{\prime T} \mathbf{P}_{K}^{T} \mathbf{P}_{K} \mathbf{1}_{K}=\mathbf{P}_{K} \mathbf{F}^{\prime T} \mathbf{P}_{K}^{T} \mathbf{1}_{K}=\left(\mathbf{P}_{K} \mathbf{F}^{\prime} \mathbf{P}_{K}^{T}\right)^{T} \mathbf{1}_{K}=\mathbf{0}_{K}
$$

where the relations $\mathbf{F}^{\prime T} \mathbf{1}_{K}=\mathbf{0}_{K}$ and $\mathbf{P}_{K} \mathbf{1}_{K}=\mathbf{1}_{K}$ are adapted. The nonnegativity of the off-diagonal entries can be easily verified by performing the multiplications in Eq. (45) $\left(i \neq j ; i, j \in \mathbb{Z}_{K}^{+}\right)$:

$$
\left\langle\mathbf{P}_{K} \mathbf{F}^{\prime} \mathbf{P}_{K}^{T}\right\rangle_{i j}=\sum_{k=1}^{K} \sum_{l=1}^{K}\left\langle\mathbf{P}_{K}\right\rangle_{i k} f_{k l}^{\prime}\left\langle\mathbf{P}_{K}^{T}\right\rangle_{l j}=\sum_{k=1}^{K} \sum_{l=1}^{K} \delta_{\pi(i) k} f_{k l}^{\prime} \delta_{\pi(j) l}=f_{\pi(i) \pi(j)}^{\prime} \geq 0
$$

where the permutation $\pi: \mathbb{Z}_{K}^{+} \leftrightarrow \mathbb{Z}_{K}^{+}$connected to the matrix $\mathbf{P}_{K}$ is introduced and the inequality $f_{\pi(i) \pi(j)}^{\prime} \geq 0$ is applied because $\pi(i) \neq \pi(j)$ in case of $i \neq j$. Choosing a suitable permutation matrix $\mathbf{P}_{K}$, we can get the Frobenius normal form of matrix $\mathbf{F}^{\prime}$ :

$$
\mathbf{P}_{K} \mathbf{F}^{\prime} \mathbf{P}_{K}^{T}=\left(\begin{array}{cccc}
\mathcal{F}_{11}^{\prime} & & & \\
\mathcal{F}_{21}^{\prime} & \mathcal{F}_{22}^{\prime} & & \\
\vdots & \vdots & \ddots & \\
\mathcal{F}_{N_{T} 1}^{\prime} & \mathcal{F}_{N_{T} 2}^{\prime} \ldots & \mathcal{F}_{N_{T} N_{T}}^{\prime}
\end{array}\right)
$$

where $\mathcal{F}_{i i}^{\prime} \in \mathbb{R}^{Z_{i} \times Z_{i}}, Z_{i}>1\left(i \in \mathbb{Z}_{N_{T}}^{+}\right)$and $N_{T} \geq 1$ is the number of the diagonal blocks in Eq. (48) which is equal to the number of the strongly connected components in the reaction graph associated to the network $\left\langle\mathbf{D}^{\prime}, \mathbf{G}^{\prime}, \mathbf{A}^{\prime}, \mathbf{k}^{\prime}\right\rangle$ [17]. The Frobenius normal form of the matrix $\mathbf{F}$ is also generated by the matrix $\mathbf{P}_{K}\left(\mathcal{F}_{i i} \in \mathbb{R}^{Z_{i} \times Z_{i}} ; i \in \mathbb{Z}_{N_{T}}^{+}\right)$:

$$
\mathbf{P}_{K} \mathbf{F P}_{K}^{T}=\left(\begin{array}{cccc}
\mathcal{F}_{11} & & & \\
\mathcal{F}_{21} & \mathcal{F}_{22} & & \\
\vdots & \vdots & \ddots & \\
\mathcal{F}_{N_{T} 1} & \mathcal{F}_{N_{T} 2} & \ldots & \mathcal{F}_{N_{T} N_{T}}
\end{array}\right)
$$


As the matrix $\mathbf{P}_{K} \mathbf{F}^{\prime} \mathbf{P}_{K}^{T}$ is Metzlerian, so $\mathcal{F}_{j i}^{\prime} \in[0, \infty)^{Z_{j} \times Z_{i}}\left(i<j ; i, j \in \mathbb{Z}_{N_{T}}^{+}\right)$and Taussky's theorem holds for the irreducible matrices $\mathcal{F}_{i i}^{\prime}\left(\operatorname{det}\left(\mathcal{F}_{i i}^{\prime}\right)=0 \Leftrightarrow \mathcal{F}^{\prime T}{ }_{i i} \mathbf{1}_{Z_{i}}=\right.$ $\mathbf{0}_{Z_{i}}$ ). Let us suppose that $\operatorname{det}\left(\mathcal{F}_{i i}^{\prime}\right)=0$ for some $i \in \mathbb{Z}_{N_{T}}^{+}$. In this case, Eq. (46) involves the subsequent connection:

$$
\sum_{j=i}^{N_{T}} \mathcal{F}^{\prime T}{ }_{j i} \mathbf{1}_{Z_{j}}=\mathcal{F}^{\prime}{ }_{i i}^{T} \mathbf{1}_{Z_{i}}+\sum_{j=i+1}^{N_{T}} \mathcal{F}^{\prime T}{ }_{j i} \mathbf{1}_{Z_{j}}=\sum_{j=i+1}^{N_{T}} \mathcal{F}_{j i}{ }_{j i} \mathbf{1}_{Z_{j}}=\mathbf{0}_{Z_{i}}
$$

which involves the relations $\mathcal{F}_{j i}^{\prime}=\mathbf{0}_{Z_{j} \times Z_{i}}\left(i<j ; i, j \in \mathbb{Z}_{N_{T}}^{+}\right) . \mathcal{F}_{i i}^{\prime}$ is called terminal if $\operatorname{det}\left(\mathcal{F}_{i i}^{\prime}\right)=0$, otherwise it is nonterminal. (These notions are analogous to the ones of the terminal and nonterminal strongly connected components from [35].) If the diagonal block $\mathcal{F}_{i i}^{\prime}$ is terminal, then zero is a simple eigenvalue, namely, $\mathcal{F}_{i i}^{\prime}$ obeys Hearon's theorem. Put it another way, $\mathcal{F}_{i i}^{\prime}$ is singular with a simple zero eigenvalue iff it is a terminal block. Similar results are achieved by Foster [19], but in a slightly more complicated way.

The singularity of the blocks $\mathcal{F}_{i i}^{\prime}$ can be extended to the blocks $\mathcal{F}_{i i}$ which case is generally not treated in the literature. If the matrix $\mathcal{F}_{i i}^{\prime}$ is terminal, then $\operatorname{det}\left(\mathcal{F}_{i i}\right)=0$ and $\mathcal{F}_{j i}=\mathbf{0}_{Z_{j} \times Z_{i}}\left(i<j ; i, j \in \mathbb{Z}_{N_{T}}^{+}\right.$), involving that the multiplicity of the zero eigenvalue in the matrix $\mathbf{F}$ is equal to the number of the terminal blocks in its Frobenius normal form.

It is well-known from the Abel-Ruffini theorem that the roots of the characteristic polynomial associated to the matrix $\mathcal{F}_{i i}\left(i \in \mathbb{Z}_{N_{T}}^{+}\right)$can be symbolically expressed in general case iff its degree is not greater than four. Consequently, the eigenvalues of an arbitrary matrix $\mathbf{F}$ can be given by closed formulas if $Z_{i} \leq 4$ or $Z_{i} \leq 5$ and $\operatorname{det}\left(\mathcal{F}_{i i}\right)=0\left(i \in \mathbb{Z}_{N_{T}}^{+}\right)$.

If either of the conditions above is in force for some block $\mathcal{F}_{i i}$, then it is advantageous to find its eigenvalues with the (linear, quadratic, cubic and quartic) root formulas. This is due to the fact that the convergence of the numerical eigenvalue algorithms is not guaranteed in certain cases. (These methods may exhibit oscillating or chaotic behavior.)

\section{Simulation}

As a real chemical application, an industrially important ten-component network, the mechanism for reductive dechlorination and elimination of chlorinated ethene system [15] is modeled (Fig. 1) whose species have algebraically closed concentration functions. In this network, denoted by $\mathcal{N}$, the reactions can be treated as formal isomerization processes whose rate coefficients are experimentally determined in aqueous solution on zinc catalysts with a specific surface area of $1.0 \mathrm{~m}^{2} \mathrm{~cm}^{-3}$ [15]. The values 


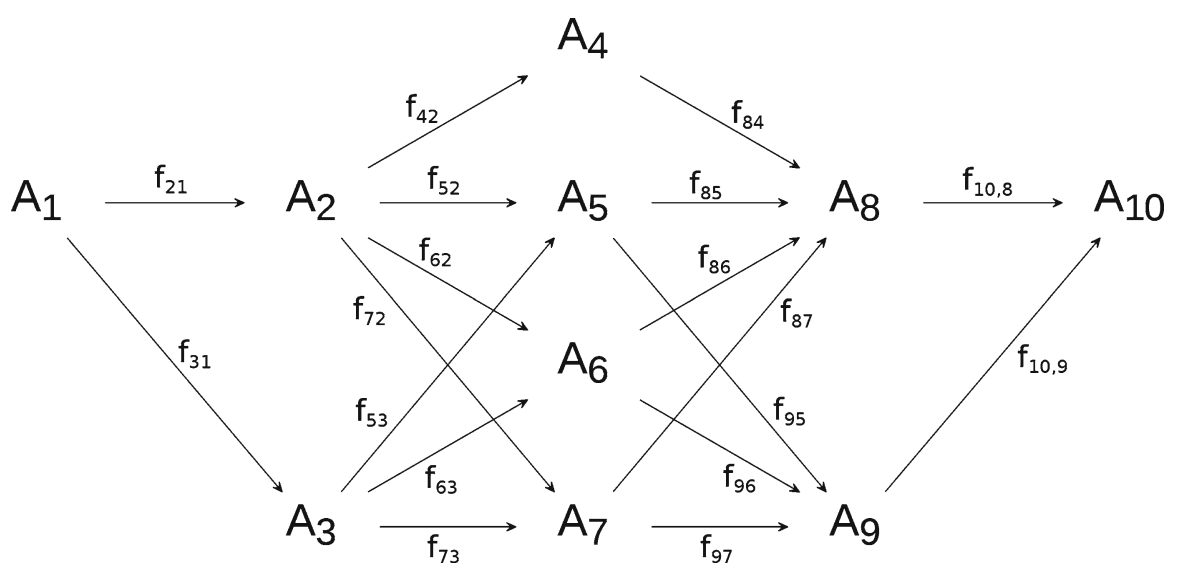

Fig. 1 Mechanism for reductive dechlorination and elimination of chlorinated ethene system [15] (Notations $A_{1}$ : tetrachloroethene, $A_{2}$ : trichloroethene, $A_{3}$ : dichloroacetylene, $A_{4}: 1,1$-dichloroe-thene, $A_{5}$ : $Z$-1,2-dichloroethene, $A_{6}: E$-1,2-dichloroethene, $A_{7}$ : chloroacetylene, $A_{8}$ : vinyl-chloride, $A_{9}$ : acetylene, $A_{10}$ : ethene)

of the rate coefficients in $\mathrm{h}^{-1}$ unit can be found in the coefficient matrix of the network $\mathcal{N}$ :

$$
\mathbf{F}=\left(\begin{array}{cccccccccc}
-305 & 0 & 0 & 0 & 0 & 0 & 0 & 0 & 0 & 0 \\
260 & -3.0841 & 0 & 0 & 0 & 0 & 0 & 0 & 0 & 0 \\
45 & 0 & -25070 & 0 & 0 & 0 & 0 & 0 & 0 & 0 \\
0 & 0.0681 & 0 & -0.0407 & 0 & 0 & 0 & 0 & 0 & 0 \\
0 & 0.603 & 0 & 0 & -0.0035 & 0 & 0 & 0 & 0 & 0 \\
0 & 1.47 & 20640 & 0 & 0 & -0.01324 & 0 & 0 & 0 & 0 \\
0 & 0.943 & 4430 & 0 & 0 & 0 & -7030 & 0 & 0 & 0 \\
0 & 0 & 0 & 0.0407 & 0.00052 & 0.00064 & 530 & -0.101 & 0 & 0 \\
0 & 0 & 0 & 0 & 0.00298 & 0.0126 & 6500 & 0 & -0.502 & 0 \\
0 & 0 & 0 & 0 & 0 & 0 & 0 & 0.101 & 0.502 & 0
\end{array}\right) .
$$

It is worth emphasizing that the coefficient of the reaction $A_{3} \rightarrow A_{5}$ is defined as zero in the study by Eykholt [15]. This value is certainly incorrect but in absence of any further information we have to apply the relation $f_{53}=0$. It can also be seen that $\mathbf{F}$ is triangular, therefore $\lambda_{i}=f_{i i}\left(i \in \mathbb{Z}_{10}^{+}\right)$. Furthermore, Eq. (51) is the Frobenius normal form of the matrix $\mathbf{F}$ whose blocks are of size $1 \times 1$.

Employing Eqs. (16)-(18), next we reproduce Fig. 5 in Ref. [15] which illustrates the analytical and numerical solution of the FLSODE related to the network $\mathcal{N}$ with the initial condition $c_{0 j}=\delta_{1 j} \mathrm{mmol} \mathrm{dm}^{-3}\left(j \in \mathbb{Z}_{10}^{+}\right)$. Knowing the initial value vector $\mathbf{C}_{0}$ and the eigenvalues, $\mathbf{F}_{\mathbf{C}_{0}}, \mathbf{E}(t)$ and $\mathbf{V}$ can be easily constructed $\left(i, j \in \mathbb{Z}_{10}^{+}\right)$:

$$
\begin{aligned}
\mathbf{F}_{\mathbf{C}_{0}} & =\left\{\left\langle\mathbf{F}^{j-1} \mathbf{C}_{0}\right\rangle_{i}\right\}, \\
\mathbf{E}(t) & =\left\{e^{\lambda_{i} t}\right\},
\end{aligned}
$$




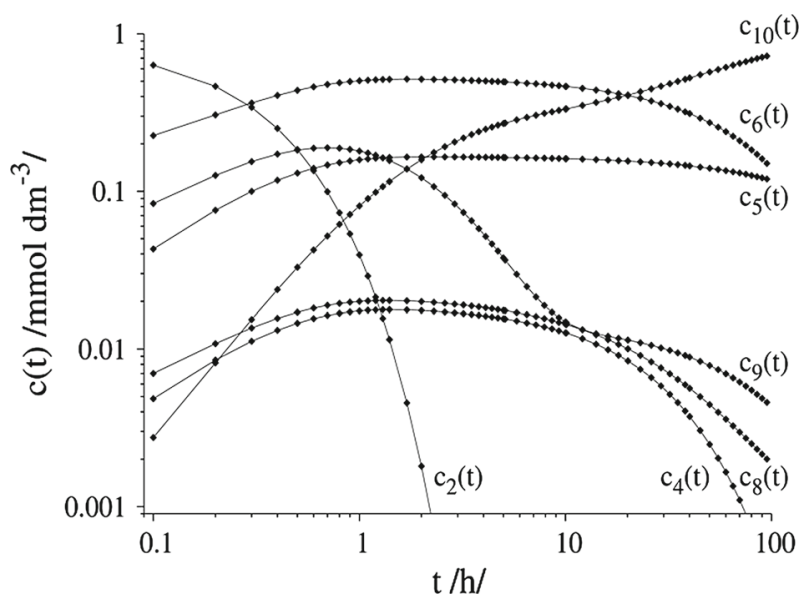

Fig. 2 Kinetic curves of the isomerization network presented in Fig. 1

$$
\mathbf{V}=\left\{\lambda_{j}^{i-1}\right\}
$$

Based on Eq. (15), these quantities do provide the solution vector $\mathbf{C}(t)=\left\{c_{i}(t)\right\}$. The calculated concentration profiles are depicted in Fig. 2 which are precisely identical to the curves of Fig. 5 in Ref. [15].

\section{Conclusions}

In this paper, ignoring any graph-theoretic concepts, an algebraic investigation of $F C R N$ s was presented. The connectedness of $C R N \mathrm{~s}$ and its impact on the coefficient matrix of FCRNs were studied. The reducibility of the coefficient matrix was interpreted via mass incompatibility which is easily comprehensible for chemists. It was shown that each conservative $F C R N$ was linearly conjugate to an $I R N$ referred to as the marker network in the paper. The marker network describes the kinetic behavior of the original network in a less complicated way. This fact was greatly utilized in the characterization of the zero eigenvalue of the coefficient matrix of FCRNs. Future work is planned to set up a linear model for the dynamics of $C R N$ s containing second and higher order reactions which is capable of insuring the nonnegativity of the concentrations.

\section{References}

1. D.H. Anderson, Math. Biosci. 71, 105 (1984)

2. D. Angeli, P. De Leenheer, E.D. Sontag, Math. Biosci. 210, 598 (2007)

3. D.S. Bernstein, Matrix Mathematics: Theory, Facts, and Formulas (Princeton University Press, Princeton, 2009)

4. D.S. Bernstein, S.P. Bhat, J. Mech. Des. 117, 145 (1995)

5. D.S. Bernstein, S.P. Bhat, Proceedings of the 38th IEEE Conference on Decision and Control, Arizona, 2206 (1999) 
6. F. Bullo, J. Cortés, Distributed Control of Robotic Networks: A Mathematical Approach to Motion Coordination Algorithms (Princeton University Press, Princeton, 2009)

7. V. Chellaboina, S.P. Bhat, W.M. Haddad, D.S. Bernstein, Control Syst. 29, 60 (2009)

8. C. Cobelli, A. Lepschy, G.R. Jacur, Math. Biosci. 44, 1 (1979)

9. C. Cobelli, A. Rescigno, IEEE T. Bio-Med. Eng. 3, 294 (1978)

10. G. Craciun, Toric Differential Inclusions and a Proof of the Global Attractor Conjecture, arXiv:1501.02860. Accessed 02 Feb 2016

11. G. Craciun, M. Feinberg, SIAM J. Appl. Math. 65, 1526 (2005)

12. G. Craciun, M. Feinberg, SIAM J. Appl. Math. 66, 1321 (2006)

13. G. Craciun, C. Pantea, J. Math. Chem. 44, 244 (2008)

14. P. Érdi, J. Tóth, Mathematical Models of Chemical Reactions: Theory and Applications of Deterministic and Stochastic Models (Manchester University Press, Manchester, 1989)

15. G.R. Eykholt, Water Res. 33, 814 (1999)

16. M. Feinberg, Arch. Ration. Mech. Anal. 46, 1 (1972)

17. M. Feinberg, Chem. Eng. Sci. 42, 2229 (1987)

18. M. Feinberg, F.J. Horn, Arch. Ration. Mech. Anal. 66, 83 (1977)

19. D.M. Foster, J.A. Jacquez, Math. Biosci. 26, 89 (1975)

20. W.M. Haddad, V. Chellaboina, Nonlinear Anal. Real World Appl. 6, 35 (2005)

21. T. Hawkins, Mathematics of Frobenius in Context (Springer, Berlin, 2015)

22. J.Z. Hearon, Bull. Math. Biophys. 15, 121 (1953)

23. J.Z. Hearon, Ann. N. Y. Acad. Sci. 108, 36 (1963)

24. D. Himmelblau, C. Jones, K. Bischoff, Ind. Eng. Chem. Fund. 6, 539 (1967)

25. F. Horn, R. Jackson, Arch. Ration. Mech. Anal. 47, 81 (1972)

26. M.D. Johnston, D. Siegel, J. Math. Chem. 49, 1263 (2011)

27. M.D. Johnston, D. Siegel, G. Szederkényi, J. Math. Chem. 50, 274 (2012)

28. T. Kailath, Linear Systems (Prentice-Hall, Englewood Cliffs, 1980)

29. G. Ladde, Math. Biosci. 30, 1 (1976)

30. G. Lente, J. Chem. Phys. 137, 164101 (2012)

31. G. Lente, Deterministic Kinetics in Chemistry and Systems Biology: The Dynamics of Complex Reaction Networks (Springer, Berlin, 2015)

32. U. Luther, K. Rost, Electron. Trans. Numer. Anal. 18, 91 (2004)

33. J.G. McWilliams, D.H. Anderson, Math. Biosci. 77, 287 (1985)

34. M. Milanese, N. Sorrentino, Int. J. Control 28, 71 (1978)

35. I. Mirzaev, J. Gunawardena, Bull. Math. Biol. 75, 2118 (2013)

36. C. Pantea, SIAM J. Math. Anal. 44, 1636 (2012)

37. L. Pogliani, M.N. Berberan-Santos, J.M. Martinho, J. Math. Chem. 20, 193 (1996)

38. L.S. Pontryagin, Ordinary Differential Equations. Translated from the Russian by Leonas Kacinskas and Walter B. Counts (Addison-Wesley Publishing Company, Reading, 1962)

39. A. Pothen, C.J. Fan, ACMT Math. Softw. 16, 303 (1990)

40. S. Schuster, R. Schuster, J. Math. Chem. 6, 17 (1991)

41. G. Shinar, M. Feinberg, Science 327, 1389 (2010)

42. O. Taussky, Am. Math. Mon. 56, 672 (1949)

43. R. Tóbiás, G. Tasi, J. Math. Chem. 54, 85 (2016)

44. A.I. Volpert, Math. USSR Sb. (English) 17, 571 (1972) 